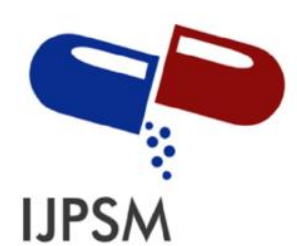

Nikam Supriya S. et al, International Journal of Pharmaceutical Sciences and Medicine (IJPSM), Vol.6 Issue. 7, July- 2021, pg. 81-101

ISSN: 2519-9889

Impact Factor: 3.426

\title{
NANOSTRUCTURED LIPID CARRIERS: A PROMISING APPROACH FOR TOPICAL DRUG DELIVERY SYSTEM
}

\author{
Nikam Supriya S. ${ }^{{ }^{*}}$; Phadatare Priya P. ${ }^{1}$; Watode Ankita B. $^{2}$; Kalyani Kayande ${ }^{3}$ \\ ${ }^{1}$ Sinhgad Institute of Pharmacy Narhe, Pune, India \\ E-mail address- priyaphadatare07@gmail.com \\ DOI: 10.47760/ijpsm.2021.v06i07.007
}

\begin{abstract}
Nanostructured lipid carriers (NLCs) are innovative pharmaceutical formulations made up of physiological and biocompatible lipids, as well as surfactants and co-surfactant. The initial generation of lipid nanoparticles was Solid Lipid Nanoparticles (SLN), which had a longer-lasting activity and was better, suited to drug penetration. The NLC is a second-generation lipid nanoparticle designed to alleviate the limitations of SLN, such as limited drug loading capacity and solid lipid polymorphism. Many benefits of topical medication delivery include avoiding first-pass metabolism, focusing active components for a local effect, and patient compliance. When compared to typical topical dose forms, nanoparticles have a greater effect in transporting medications through the skin. The structure, composition, many formulation methods, and characterization of NLCs are all important aspects in formulating a stable drug delivery system, as discussed in this review paper. A variety of approaches are used to make solid lipid nanoparticles and nanostructured lipid carriers, which are discussed in this paper. Lipid nanoparticles have a variety of features that make them suitable for topical usage in cosmetics and medicinal formulations. Because of extensive positive benefits such as skin hydration, skin occlusion, and skin targeting, NLCs have a significant potential in the pharmaceutical market. Skin hydration is important for API topical distribution because it hydrates the skin, which causes the pores to open. Trans epidermal water loss decreases due to the occlusion nature of lipid nanoparticles, softening the skin. It is more suited since it uses biodegradable grade lipid, which does not cause toxicity like polymeric Nano formulations. Actually, because of their biodegradable composition, NLCs are a "Nano safe" carrier that has a lot of potential for overcoming the obstacles of topical distribution.
\end{abstract}

Keywords: Topical drug delivery, Skin, Nanostructured Lipid Carriers (NLC). 


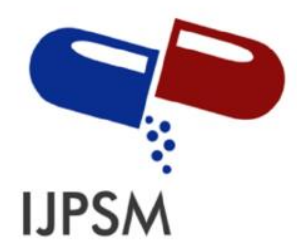

Nikam Supriya S. et al, International Journal of Pharmaceutical Sciences and Medicine (IJPSM), Vol.6 Issue. 7, July- 2021, pg. 81-101

ISSN: 2519-9889

Impact Factor: 3.426

\section{Introduction to topical drug delivery ${ }^{[1][2][3][4]}$}

The oral route is the most common and effective way of drug administration. Unfortunately, oral drug delivery systems have a number of significant drawbacks, including drug degradation in the gastrointestinal tract (GIT), pre-systemic metabolism, and severe side effects. The topical drug delivery system is one way that could solve the issues associated with the oral route. The first transdermal therapeutic device, in the form of a patch containing scopolamine, was created in 1981 for the treatment of motion sickness. Because skin acts as a natural and protective barrier; topical medication administration is extremely difficult. Transdermal drug delivery has a higher rate of patient compliance than alternative, more unpleasant approaches that involve a needle injection. Several topical drug delivery techniques are currently being explored, including microneedle injection, chemical penetration enhancers, and physical barrier breakdown by ultrasound, and Nano carriers. Topical delivery, which transports medication molecules into the systemic circulation via skin layers, is mostly utilized in the treatment of local skin problems. The topical route of drug administration has several advantages, including first-pass metabolism, no systemic drug absorption, ease of sustained drug delivery, and the ability to continue a therapeutic dosing regimen while using active compounds with short biological halflives. However, distinct issues arise when it comes to managing drug bioavailability at various skin target areas, making topical drug administration a challenge.

\section{Structure of skin ${ }^{[5][6][7][8]}$}

Topical medication delivery using nanotechnology has opened up a new arena in the delivery of pharmaceuticals through the skin. With an average surface area of $1.8 \mathrm{~m}^{2}$; the skin is the biggest organ in the human body. The skin serves a variety of purposes, including protecting the body from external contaminants. The epidermis, dermis, and hypodermis (subcutaneous layer) are the three layers of human skin. Figure 6 depicts the structure of human skin (1)

\section{1) Epidermis}

The epidermis is a large organ in the human body. It takes up 1.5-2 $\mathrm{m}^{2}$ in adulthood. The Stratum Corneum (SC) is the epidermis' top layer. The stratum corneum is the epidermis' top layer, with a thickness of 10-40 m. It serves as a primary physical barrier to dehydration, infection, chemicals, and mechanical stress. The stratum corneum is made up of 15 to 20 layers of dead corneocytes that lie parallel to the skin's surface, and it improves and deteriorates throughout time due to cell proliferation and exfoliation. Michaels et al proved that certain medications have better permeability than others, despite the fact that it has an inherent barrier function against foreign molecules. The molecular mass $(500 \mathrm{Da})$ and lipophilicity of these medicines are similar. Big 


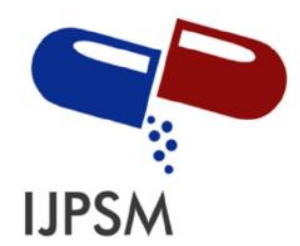

Nikam Supriya S. et al, International Journal of Pharmaceutical Sciences and Medicine (IJPSM), Vol.6 Issue. 7, July- 2021, pg. 81-101

ISSN: 2519-9889

Impact Factor: 3.426

molecules take longer to diffuse, making efficient delivery of large hydrophilic medicines a major problem in topical drug delivery.

\section{2) Dermis}

The dermis is the second layer underneath the epidermis. Fibroblasts, cutaneous dendritic cells, and mast cells are all present. The dermis layer is a supporting matrix made up of connective tissue. Polysaccharides and proteins, such as glycosaminoglycan and proteoglycan macromolecules, are found in the dermis, which serve to keep the skin hydrated. It also contains collagen and elastin protein fibres, which give skin its tensile strength, suppleness, and pliability.

\section{3) Hypodermis}

Subcutaneous tissue is another name for hypodermis. It is the skin's thickest layer, where half of the body fat is stored. It is made up of loose, whitish, fibrous connective tissue that is cushioned by fat. The dermis and hypodermis layers contain blood arteries, collagen fibres, lymphatic, nerve cells, and skin appendages.

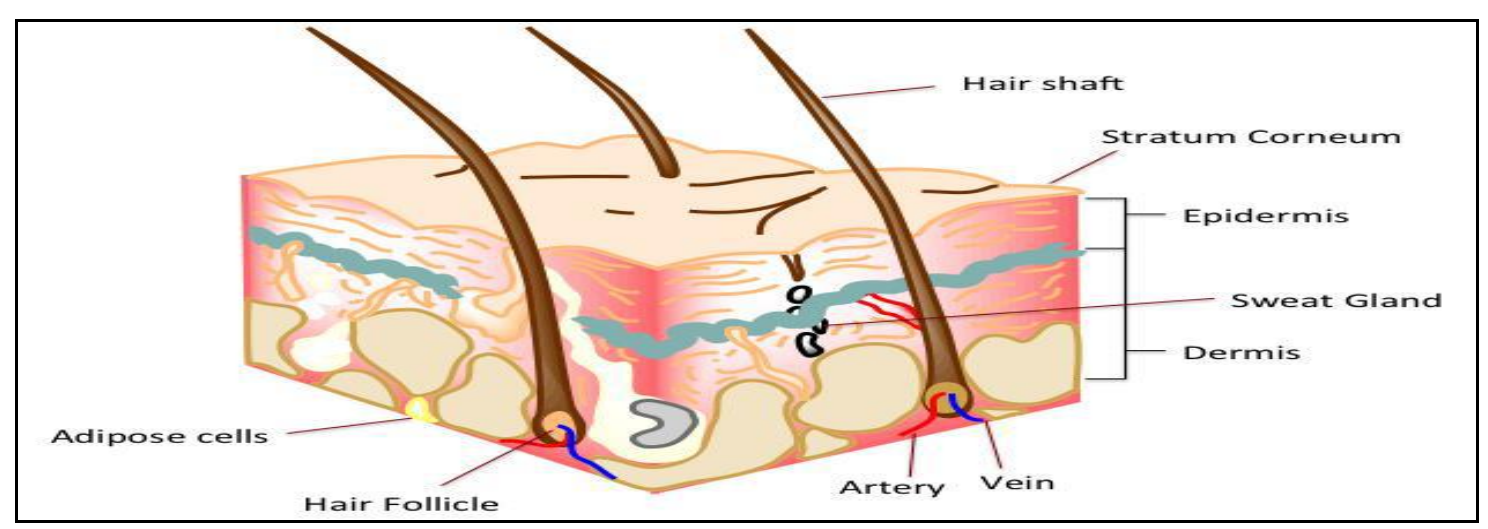

Figure.1 Structure of human skin

\section{Advantages of topical drug delivery ${ }^{[9][10]}$}

1. In transdermal medication distribution, it is feasible to avoid first-pass metabolism.

2. It also reduces the risk of gastric incompatibility.

3. Drug distribution by transdermal means is more targeted to a specific area.

4. It also boosts patient compliance. 


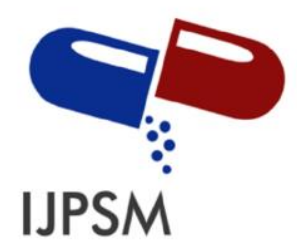

Nikam Supriya S. et al, International Journal of Pharmaceutical Sciences and Medicine (IJPSM), Vol.6 Issue. 7, July- 2021, pg. 81-101

5. It is appropriate for self-medication.

6. It allows for the use of drugs with a short biological half-life and a limited therapeutic window.

7. Transdermal drug administration allows you to effortlessly stop taking medication when you need to. ${ }^{9}$

Lipid nanoparticles are colloidal carriers that were developed in the preceding decade as a replacement for traditional carriers such as emulsions, liposomes, and polymeric nanoparticles. Solid Lipid Nanoparticles (SLN) and Nanostructured Lipid Carrier (NLC) are two forms of lipid nanoparticles that have piqued the curiosity of formulator's worldwide.

\section{Introduction to Nanostructured Lipid Carriers (NLC) ${ }^{[11][12][13][14][15]}$}

Professor R. H. Muller of Germany and Professor M. Gascon of Italy studied lipid nanoparticles as drug delivery devices around the turn of the nineteenth century. These nanoparticles are made from solid lipids or a mixture of solid and liquid lipids, and they are stabilized by emulsifiers.

Solid lipid nanoparticles (SLNs) have a number of properties that make them ideal for topical administration. However, depending on the medicine, lipid polymorphism may present issues such as limited drug loading, gelation risk, and drug leakage during storage. Nanostructured lipid carriers (NLCs) have since been developed to address the limitations of solid lipid nanoparticles.

NLCs emerged as a novel medication delivery technology in the late 1990s. It's also known as lipid nanoparticles of a new generation. Nanoparticles are colloidal particles that range in size from 10 to 1000 nanometers. The modification of SLNs and a mixture of solid and liquid lipids generated by heating and cooling crystallization are used in this delivery system. NLCs have several advantages over SLNs, such as a disordered crystal structure that can assist avoid drug loading leakage and provide a higher drug payload. NLC is a biodegradable carrier material with low in vivo toxicity that may be loaded with hydrophobic or hydrophilic medicines with a wide range of drug-loading properties. Surface modification of NLCs is also possible. The NLC particles' physical and chemical properties are stable. Structure of a nanostructured lipid carrier is shown in Figure (2) 


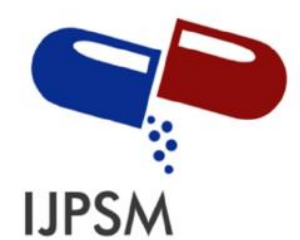

Nikam Supriya S. et al, International Journal of Pharmaceutical Sciences and Medicine (IJPSM), Vol.6 Issue. 7, July- 2021, pg. 81-101

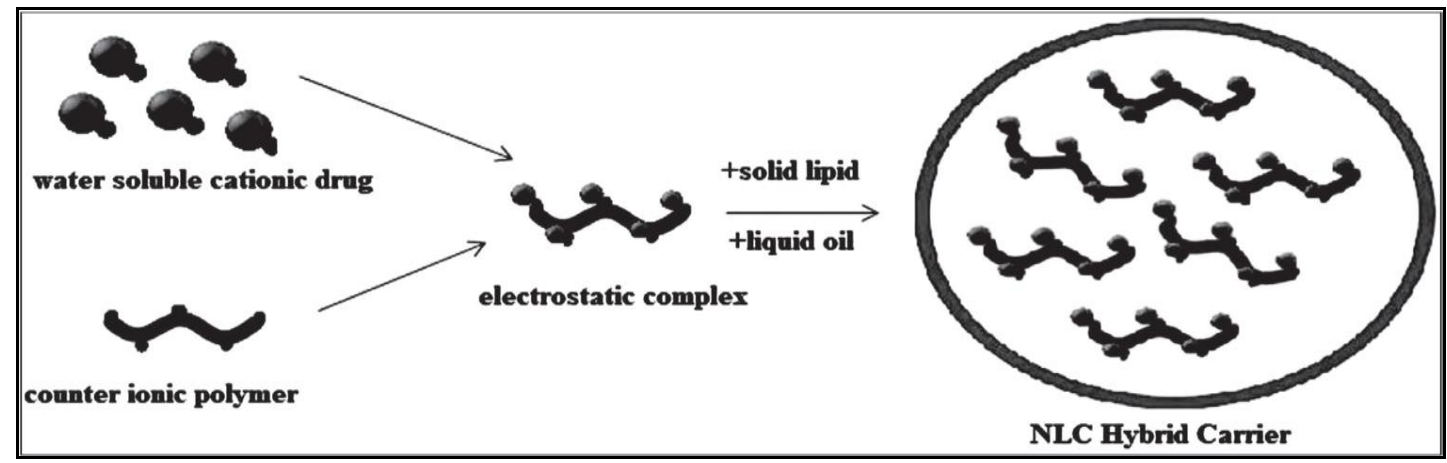

Figure.2 Structure of Nanostructured Lipid Carrier

Nanostructured lipid carriers (NLC) have been touted as viable carriers for transdermal medication administration throughout the last decade due to a number of appealing characteristics. When compared to the oral and parenteral routes, topical medication distribution is the most common mode of administration since the systemic load of the medicine and drug-related side effects are minimized.

\section{Advantages of nanostructured lipid carriers ${ }^{[16]}$}

- NLCs are easily approved by regulatory agencies and validated

- NLCs have excellent biocompatibility.

- It is a water-based technology, organic solvents can be avoided.

- NLCs are easy to scale up and sterilize, and they are less expensive than polymeric/surfactant-based carriers.

- NLCs have the potential to carry both lipophilic and hydrophilic medicines at the same time, which improves pharmaceutical stability.

- NLCs communicate substantial and greater drug content than other carriers currently on the market.

\section{Types of nanostructured lipid carriers ${ }^{[17][18]}$}

There are three distinctive types Nanostructured lipid carriers depending on nanostructure, synthesis, and proportions of solid and liquid lipids. ${ }^{17}$ 


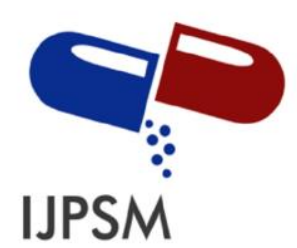

Nikam Supriya S. et al, International Journal of Pharmaceutical Sciences and Medicine (IJPSM), Vol.6 Issue. 7, July- 2021, pg. 81-101

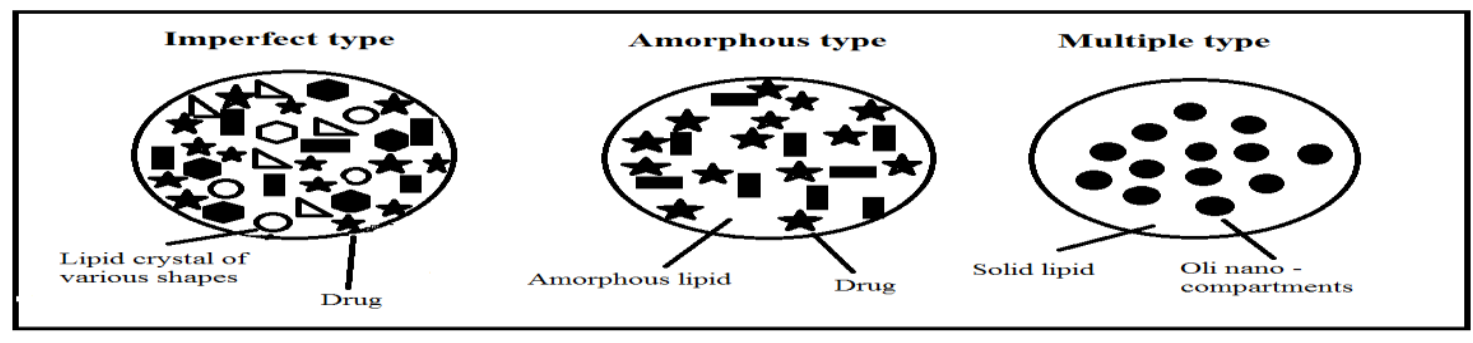

Figure. 3 (1) imperfect type (2) amorphous type (3) multiple type

\section{Imperfect type}

The liquid phase lipid content of the Imperfect kind of NLC is quite low (oil). It's made up of saturated and unsaturated lipids with varying fatty acid chain lengths, which causes flaws in the lipid matrix and drug storage compartments. During the manufacturing process of crystallization, the defective NLC is prone to drug expulsion. ${ }^{17}$

\section{Amorphous type}

The amorphous version of NLC produces a solid lipid with no crystalline structure. This type of NLC is made by combining solid and liquid lipids, such as hydroxyoctacosanyl hydroxystearate isopropylmyristate. ${ }^{17}$

\section{Multiple types}

Oil Nano compartments are coated with solid lipid and medication is dissolved in the oil compartments in several types of NLC. This form of NLC prevents drug expulsion by including a larger concentration of liquidphase lipids in the lipid matrix, after which the oil exceeds its solubility limit and precipitates into Nano compartments during the chilling process. ${ }^{18}$

\begin{tabular}{|l|l|}
\hline Ingredient & Example \\
\hline Solid lipid & $\begin{array}{l}\text { Bees wax, Carnauba wax 2442, Stearic acid, Palmitic acid, Compritol@888 ATO, } \\
\text { Precirol®ATO5 }\end{array}$ \\
\hline Liquid lipid & $\begin{array}{l}\text { Miglyol } ₫ 812, \text { Castor oil, Oleic acid, Linoleic acid, Palm oil, Olive oil, Argon oil, } \\
\text { Coconut oil, Cetiol V }\end{array}$ \\
\hline $\begin{array}{l}\text { Emulsifying } \\
\text { agents }\end{array}$ & $\begin{array}{l}\text { Pluronic®F68 (Poloxamer188), Pluronic®F127 (Poloxamer 407), Tween 20, Tween 40, } \\
\text { Tween 80, Gelucire®50/13, Solutol® HS15, alcohol, Sodium deoxycholate, Sodium } \\
\text { glycocholate, Sodium oleate, Soya lecithin, Phosphatidylcholines, Egg lecithin }\end{array}$ \\
\hline Counter-ion & $\begin{array}{l}\text { Sodium hexadecyl phosphate, Mono hexadecyl phosphate, Mono octyl phosphate, } \\
\text { Dextran sulphate sodium salt, Hydrolysed and polymerized expoxidised soybean oil }\end{array}$ \\
\hline
\end{tabular}

Table1. Excipients used in formulating NLCs 


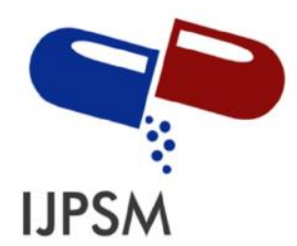

Nikam Supriya S. et al, International Journal of Pharmaceutical Sciences and Medicine (IJPSM), Vol.6 Issue. 7, July- 2021, pg. 81-101

\section{Beneficial role of NLCs in topical drug delivery ${ }^{[19][20][21][22][23][24][25][26][27][28][29][30][31][32]}$}

Since ages, the transdermal medication delivery mechanism has been used to achieve a variety of therapeutic goals on various anatomical layers of the skin (e.g. epidermis, dermis, hypodermis and surface). Nonetheless, there have been a number of issues with traditional topical therapies, such as skin barrier impermeability, limited efficacy, and excessive application frequency. The current focus of the researcher in the pharmaceutical and cosmetic sectors is partially related to creating NLC for topical and dermal use. NLCs are made up of biologically active and biodegradable lipids that are low in toxicity and have a number of beneficial properties, including adhesiveness, occlusion, skin hydration, lubrication, smoothness, emolliency, skin penetration enhancement, modified release, improved formulation appearance with a whitening effect, and active protection from degradation. The following are the primary advantages that NLC has in terms of transdermal medication delivery that make it superior.

\section{Skin permeation}

Numerous scientific papers have claimed that NLC has the ability to restrict the rate of medication penetration into the epidermis, hence limiting unwanted active absorption into the bloodstream.

The smaller size of NLCs assures a close touch with the Stratum corneum and can help the active chemical penetrate deeper into the skin. The routes of nanoparticle penetration (Figure 4) have been agreed upon by researchers. The following are suggested pathways for improved particle penetration across the stratum corneum followed by drug diffusion:

1. Flawless medication-loaded vesicles emerge in the skin's various layers.

2. Skin lipid-fluidizing and altering properties of lipid vesicles operate as penetration enhancers.

3. After circulating carrier lipids with cellular skin lipids, introduce a nanostructure lipid carrier and skin drug interchange.

4. The hair follicle, pilosebaceous, and sweat gland pores make up the appendageal pathway.

The hair follicle is responsible for an invagination of the epidermis that extends deep into the dermis, resulting in a more notable accessible region for drug absorption. Between the appendageal channels, the hair follicle has become the most important penetration pathway for nanoparticles. Furthermore, the hair follicles serve as a physical storage location for topically applied nanoparticles, which are often drawn deep into the tissue, up to $2000 \mathrm{~m}$. The increased storage volume of hair follicle casts allows for both permeation augmentation and prolonged release. Drug particles collect in follicular casts, which are tracked by drug diffusion from Nano carriers into the skin. 


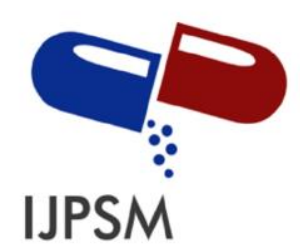

Nikam Supriya S. et al, International Journal of Pharmaceutical Sciences and Medicine (IJPSM), Vol.6 Issue. 7, July- 2021, pg. 81-101

ISSN: 2519-9889

Impact Factor: 3.426

Skin penetration of NLCs is determined by their composition as well as physicochemical qualities such as charge on particle surface, size, aggregation hydrophobicity, particle solubility in the skin, particle solubilizing properties to skin lipids, and particle film formation capacity.

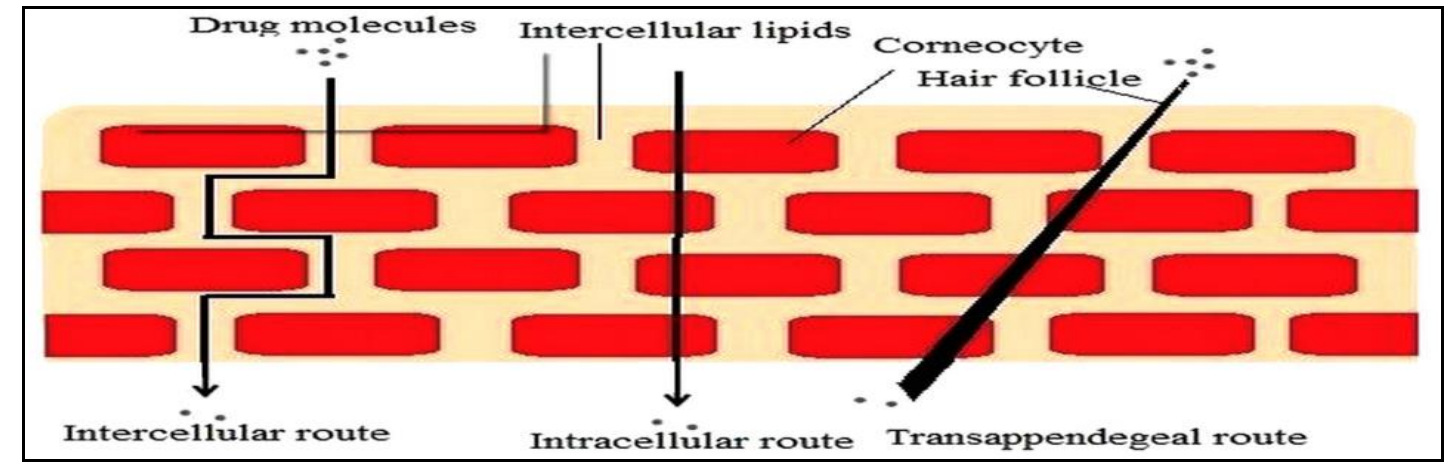

Figure.4 routes of drug penetration across the stratum corneum

\section{Trans epidermal water loss (Tewl)}

The amount of water that passively evaporates through skin to the external environment due to a water vapor pressure differential on both sides of the skin barrier is referred to as trans epidermal water loss and is used to describe skin barrier function. TEWL loss measures reveal a damaged and compromised barrier function of the stratum corneum. The stratum corneum is disrupted, and intercellular lipids are reduced, as TEWL rises. Because of enhanced skin occlusion and skin moisture, topical administration of NLC depletes TEWL. It was discovered that applying NLCs to the skin helped to reduce moisture loss as compared to untreated skin. This is due to lipid Nano carriers' ultrafine size, which activates a large surface area and improves sticky qualities. Water evaporation from the skin is prevented by the lipid particle forming a uniform compact coating over the skin. Because the particles are smaller, less water evaporates from the skin. The presence of a highly crystalline lipid in the formulation efficiently minimizes moisture loss from the stratum corneum. Emollients like lecithin and propylene glycol are used in NLC formulations to help replace depleted natural lipids in the gap between corneocytes in the stratum corneum, preventing excessive TEWL.

\section{Skin occlusion}

In general, lipid Nano carriers exhibit irregular epidermal occlusive properties, which could promote bioactive penetration into the stratum corneum by preventing water evaporation. Nanoparticles are 15 times more occlusive than micro particles, according to research. Muller et al describe NLC as an "invisible, penetrationenhancing occlusive plastic foil." 


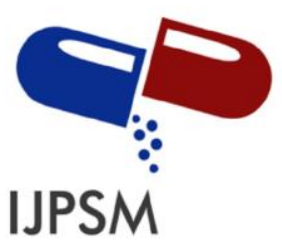

Nikam Supriya S. et al, International Journal of Pharmaceutical Sciences and Medicine (IJPSM), Vol.6 Issue. 7, July- 2021, pg. 81-101

The scope of the NLC occlusive property is determined by the following factors:

Nano carriers have a small particle size, which minimizes water evaporation from the skin. Lipid micro particles with a diameter greater than $1 \mathrm{~m}$ have a $10 \%$ occlusion factor, but lipid nanoparticles with a diameter of $200 \mathrm{~nm}$ have a 50\% occlusion factor. Wissing et al. further show that $4 \mathrm{mg}$ of topical product containing $4 \%$ lipid nanoparticles with a diameter of $200 \mathrm{~nm}$ is required to establish a monolayer film on skin. The hydrophobic nature of this occlusive coating, as seen in Figure 5, prevents skin drying, inhibits the entry of UV filters in sunscreens and other whitening effects, and provides lubrication and control release qualities.

Crystallinity and lipid concentration: The NLC formulation contains a high concentration of lipid (50-60\%), which acts as an occlusive agent. It is in charge of keeping moisture in the stratum corneum. Then it was discovered that low melting lipids and extremely crystalline particles can achieve high occlusivity. It was discovered that formulations with particles smaller than $400 \mathrm{~nm}$ and at least 35 percent lipid of high crystallinity were the most effective. The occlusive factor decreases as the oil content in the NLC recipe rises. Super cooled melts (noncrystalline nanoparticles) have no occlusive qualities; however the occlusive nature of NLCs increased skin moisture.

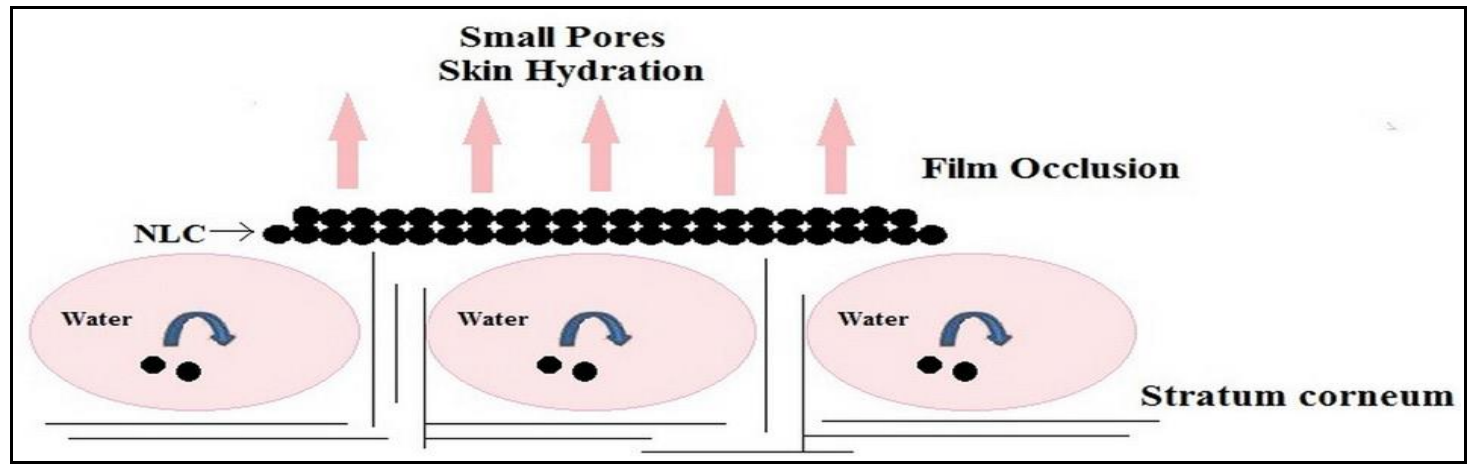

Figure.5 occlusion effect of NLC

\section{Skin hydration and elasticity}

The water content of human skin ranges from $10 \%$ to $20 \%$ of the dry weight of the tissue. The surface lipid content and moisture state are critical factors in the frictional characteristics of the skin. Acceptable skin hydration decreases the indications of ageing and protects against environmental harm. NLC made from biocompatible and physiological lipids adheres quickly to the skin and has an occlusive effect that increases skin hydration. Lipid nanoparticles also slow moisture loss, allowing the corneocyte packing to release later. The enlarged intercorneocyte gap improves medication penetration. Because of their ultrafine size, NLCs may be able to incorporate this enhanced hydration feature. Lipid nanoparticles bind to the lipid film of the stratum corneum due to their tiny size (hydrophobic interactions). The adsorbed coating repairs injured skin and, as a result, restores a thin protective film. The dimensions of the capillary channels of nanoscale pores will be 


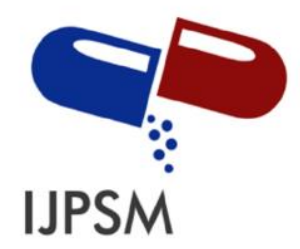

Nikam Supriya S. et al, International Journal of Pharmaceutical Sciences and Medicine (IJPSM), Vol.6 Issue. 7, July- 2021, pg. 81-101

significantly lower as a result of the smaller particle size; as a result, the hydrodynamic evaporation of water will be reduced.

According to Loo et al, a high concentration of lipids in NLCs causes the occlusive layer to become more hydrated. Less Trans epidermal water loss is aided by the sliding of more lipids into the skin's intercellular space. Humectants in the formulation help to hydrate the stratum corneum by attracting moisture from the dermis and the air. Tichota and colleagues developed an argon oil NLC-based hydrogel that had a synergistic effect on skin hydration (NLC occlusion plus argon oil hydration).

A corneometer is a device that measures the moisture of the skin. The mechanism of this instrument is to measure capacitance or conductance of a dielectric medium. The dielectric property of the stratum corneum changes as its moisture level rises. The procedure is simple, quick, and unaffected by skin residues. It has been discovered that when skin moisture is increased, the concentration of lipids increases. Skin micro relief is used to evaluate the hydration efficacy of cosmetics and can be measured using roughness, scaling, smoothing, and wrinkling metrics. A product's moisturisation impact causes an increase in skin hydration, which soothes wrinkles and improves bioactive penetration into specific skin layers. Any change in the skin's moisture level is measured using an arbitrary unit.

\section{Stability of bio actives}

One of the most important properties of lipid nanoparticles is their ability to postpone the chemical destruction of actives via photochemical, hydrolytic, and oxidative pathways. The chemical stability of medicinal molecules is strongly influenced by the lipid nanoparticles' solid lipid matrix. The chemical stability of NLC produced with less crystalline solid lipid and lattice defect was improved, according to researchers, due to a larger drug arrangement inside the lipid matrix. The exchange of actives with the aqueous phase is reduced in the solid state (diffusional law by Einstein). Similarly, the structure and chemical stability of lipids must be investigated. As a result, choosing the best appropriate lipid during preformulation trials is critical. Actives that are merged in the flaws at the less crystalline lipid matrix of NLC have been known to provide sustained physical stability in studies.

\section{Prolonged effect}

The kind of lipid (solid or oil) utilized to manufacture the vehicle, the concentration of surfactant, the solubility and concentration of active in the lipid matrix, and the process of producing NLC all influence the release profile of lipid nanoparticles. NLCs are created by combining solid lipids with spatially incompatible liquid lipids, resulting in unique nanostructures with improved drug incorporation and release capabilities. 


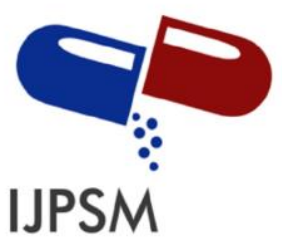

Nikam Supriya S. et al, International Journal of Pharmaceutical Sciences and Medicine (IJPSM), Vol.6 Issue. 7, July- 2021, pg. 81-101

ISSN: 2519-9889

Impact Factor: 3.426

\section{Skin targeting}

On a variety of therapy, targeted medication delivery to the epidermal layer could provide significant benefits over systemic dosing. The capacity of topical drugs to reach the intended site of action (particular skin layers) and remain there in therapeutic effective concentration for the appropriate time is hazarded in the treatment of skin problems such as acne, fungal infection, or hair loss. By creating dermal NLC, conventional topical carriers' poor therapeutic efficacy and unpleasant responses can be overcome. The method of targeting the epidermis layer is advantageous in terms of safety and a dense network of antigen-presenting cells in the epidermis, which results in a stronger immune response.

The notion that which particle feature would result in more skin targeting is still a point of contention among researchers. Different pharmacological properties such as particle carrier size, surface charge, and composition material have been discovered to alter skin penetration and pharmacodynamics of encapsulated medicines. Because of the capillary network surrounding this structure, the degree of medication interaction with plasma protein disrupts epidermal targeting and particle penetration deep into HF, especially during the growth phase. Nanoparticles can diffuse fast and continuously into the dermis or subcutaneous region after accretion in HF, enhancing epidermal targeting and local drug administration.

It was discovered that changing the size of particle carriers allows for intra- or trans-follicular penetration and drug administration (Figure 6). When particles of various sizes and morphologies are applied to the skin, they collect at the hair follicle openings and enter along the follicular duct. Because penetration depths vary depending on the kind of hair follicle and particle size, particle-based medication delivery methods can be employed to target specific locations inside the follicular duct. The movement of the hair may also help nanoparticles with a diameter of $20 \mathrm{~nm}$ penetrate. Because a high sebum/water partition coefficient is a primitive property for delivery into HF, lipids that resemble the composition of sebum may also aid follicular penetration. The disintegration or erosion of lipid particles in sebum can also indicate epidermal targeting. Surface charge manipulation can potentially be employed as a targeting strategy.

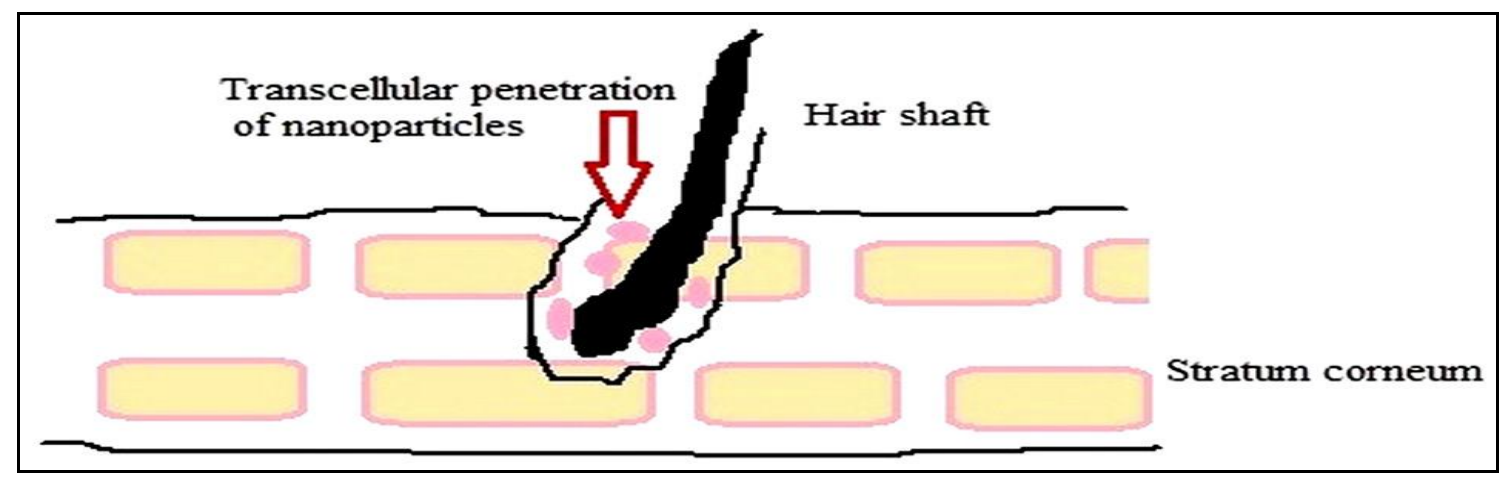

Figure.6 Trans follicular penetration of nanoparticles 


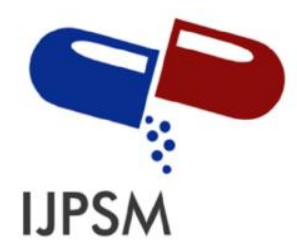

Nikam Supriya S. et al, International Journal of Pharmaceutical Sciences and Medicine (IJPSM), Vol.6 Issue. 7, July- 2021, pg. 81-101

\section{Production techniques of NLCs ${ }^{[25][26][27][29][30][31]}$}

The preparation of NLC can be done in a variety of ways, including high pressure homogenization. Solvent emulsification evaporation technique, Solvent emulsification diffusion method, Micro emulsion method, Double emulsion method, Solvent Injection method, High shear homogenization and ultra-sonication method, and so on. NLC can be made via a variety of traditional ways, but the most commonly used method is high pressure homogenization, which allows for large-scale manufacture.

\section{High pressure homogenization}

This is a dependable and robust technology for producing NLC on a commercial scale. Because of the high pressures utilized in the homogenization procedure, it is feasible to avoid using organic solvents in preparations, making them more environmentally friendly. Furthermore, high-pressure homogenization is an appealing technique that is simple to scale up. For topical use, it's also employed in the production of medications and cosmetics. Hot homogenization takes place at a high temperature, while cold homogenization takes place at a low temperature. Prior to high pressure homogenization, the active component is dissolved in molten lipid in both procedures. In the homogenizer's tiny gap, fluid travels at high pressure (100-2000 bar).

\section{Hot homogenization technique}

Homogenization is carried out at a high temperature in this method. The solid lipids are melted at a temperature greater than 5-10 degrees Celsius over their melting point. By combining liquid lipid with the medicine to be encapsulated, dispersion is created. Then, using a high shear mixing equipment, this combination is disseminated in an aqueous surfactant solution that has been heated to the same temperature. It then leads to the creation of a pre-emulsion. At a regulated temperature, the produced pre-emulsion is injected into a high-pressure homogenizer. Homogenization usually takes 3 to 5 cycles at 500-1500 bar. As the nanoemulsions cools, the lipid recrystallizes, resulting in the creation of nanoparticles. Heat sensitive components may be degraded if high temperatures are used throughout the procedure. Another issue that could develop is a decline in surfactant emulsifying capacity due to high temperatures, as surfactants have a cloud point below $85^{\circ} \mathrm{C}$. Nano carriers may become unstable as a result of this.

\section{Cold homogenization technique}

Cold homogenization is a process in which a lipid melt contains an active agent is rapidly chilled to solidify using liquid nitrogen or dry ice, then milled and ground before being disseminated in a cold surfactant phase and homogenized at room temperature. In cold homogenization, a high pressure of 5-10 cycles of $1500 \mathrm{bar}$ is usually employed. This approach reduces drug temperature exposure and is particularly well suited to thermo labile medicines. Other advantages of this approach include increased drug entrapment efficiency and consistent drug dispersion within the lipid. It does, however, result in nanoparticles with uneven shapes. 


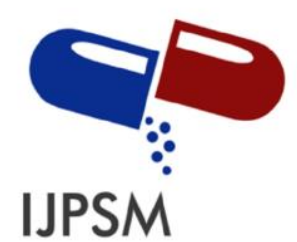

Nikam Supriya S. et al, International Journal of Pharmaceutical Sciences and Medicine (IJPSM), Vol.6 Issue. 7, July- 2021, pg. 81-101

ISSN: 2519-9889

Impact Factor: 3.426

\section{Solvent-emulsification evaporation method}

The lipids (solid lipid + liquid lipid) and medication are dissolved in a water immiscible organic solvent in the solvent-emulsification evaporation process (cyclohexane, chloroform). The resulting mixture is disseminated in an aqueous emulsifier solution, resulting in an o/w emulsion. To remove the solvent from the emulsion, evaporation under lower pressure is used. This evaporation causes nanoparticles to disperse in the aqueous phase (by lipid precipitation in the aqueous medium). This approach avoids any thermal stress, although it does have one drawback: it requires the use of an organic solvent. Depending on the solid lipid and surfactant, particle size can range from $30-100 \mathrm{~nm}$

\section{Solvent-emulsification diffusion method}

To maintain initial thermodynamic equilibrium, the solvent and water are both saturated in this approach. The medication and the lipids are then dissolved in a water-saturated solvent. To make an o/w emulsion, lipids and medication are emulsified in a solvent-saturated aqueous emulsifier solution with the use of a homogenizer. Diffusion of the organic solvent from the emulsion droplets to the continuous phase causes the lipid nanoparticles to precipitate after dilution with excess water (ratio: 1:5-1:10). Ultrafiltration or lyophilisation can then be used to remove the solvent. Diffusion of solvents has progressed. When compared to volatile solvents, the majority of the solvents used have a superior safety profile.

\section{Micro emulsion method}

The solid lipid is melted first, followed by the addition of liquid lipid and the solubilisation of the medication in the resulting mixture. A mixture of emulsifier, co-emulsifier, and water is heated to the same temperature one by one. The lipid and aqueous phases are combined in the correct proportions. Then, by gently churning it, thermodynamically stable oil in water hot micro emulsion is created. The size of the nanoparticles is determined by the micro emulsion droplet size as well as the temperature differential between the micro emulsion and the ice water. Particle aggregation can be avoided by rapid cooling and thereby solidification. Then, as a result, smaller particles are produced. Because the NLC dispersions produced by this method had a substantial number of particles in the micron range, the period of stirring, percentage of lipids, and amount of medication were tuned under this condition to achieve the desired size and entrapment efficiency. Because this process does not require any extra equipment or energy to produce NLC, commercializing the technique is simple.

\section{Double emulsion technique}

This method is mostly utilized to create lipid nanoparticles that are loaded with hydrophilic medicines. This approach, as seen in the micro emulsion method, overcomes the problem of water soluble moiety separation in the aqueous phase from the oily phase. To make a primary emulsion (w/o), the drug is first dissolved in an aqueous solvent (inner aqueous phase) and then dispersed in a lipid phase (molten solid lipid + liquid lipid+ 


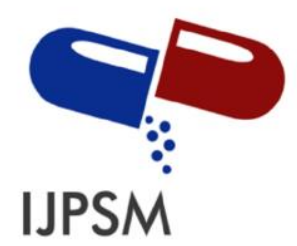

Nikam Supriya S. et al, International Journal of Pharmaceutical Sciences and Medicine (IJPSM), Vol.6 Issue. 7, July- 2021, pg. 81-101

lipophilic surfactant+ lipophilic active moiety). Both the lipid and aqueous phases are kept at the same temperature. The stabilizer prevents drug loss to the external phase during solvent evaporation. The primary emulsion is then mixed in a large volume of surfactant aqueous solution and sonicated to create a double emulsion (w/o/w). Lipid nanoparticles are then purified using ultrafiltration or solvent evaporation.

\section{Solvent injection technique}

It is a viable new method for producing lipid nanoparticles. Lipids are dissolved in a water-miscible solvent (such as ethanol, methanol, acetone, or isopropyl alcohol) or a water-soluble solvent mixture in this procedure. The mixture is then swiftly injected into an aqueous surfactant solution while being constantly stirred. The dispersion is then filtered to remove any excess lipid. The approach is based on rapid solvent diffusion across the solvent-lipid interface with the aqueous phase. The diffusion rate of the organic solvent through the solvent-lipid interface determines the particle size of Nano carriers. This technique has several advantages, including ease of use, efficiency, variety, the absence of technical equipment (e.g., a high-pressure homogenizer), and the use of permitted organic solvents.

\section{High shear homogenization and ultra-sonication}

One of the procedures for producing NLCs is high shear homogenization or ultra-sonication. Devices are used in these dispersion procedures to prepare Nano carriers. Nano dispersion is formed when solid and liquid lipids are melted and dispersed in an aqueous surfactant solution during high shear homogenization or ultrasonication. Ultrasonic cavitation, which generates violently and asymmetrically collapsing vacuum bubbles and breaks apart particles down to the nanoscale scale, creates the intense shear forces required for nanoemulsification.

Homogenization, emulsification, deagglomeration, dispersion, and milling are all favoured results of probetype ultra-sonication. The kind and quantity of lipid and surfactant, their ratio, sonication or agitation time, and speed are some of the parameters that must be tuned in order to obtain a repeatable technique that produces smaller Nano carriers. Low dispersion quality is a drawback of high shear homogenization and ultrasonication. Furthermore, metal contamination from the equipment is a major issue with ultra-sonication.

Characterization of NLCs ${ }^{[32][33][34][35]}$

\section{Particle size}

Photon Correlation Spectroscopy is used to determine particle size (PCS). Dynamic light scattering is another name for it. In comparison, it is a sensitive and precise approach. PCS can detect particles as small as $3 \mathrm{~m}$. In practice, the device calculates the average particle size (z-average) using the intensity of scattered light and the angle of light scattering. Particle sizes smaller than $400 \mathrm{~m}$ are usually favored since they exhibit adequate penetration. Particle size is highly influenced by surfactant concentration and type. Particle size reduces as 


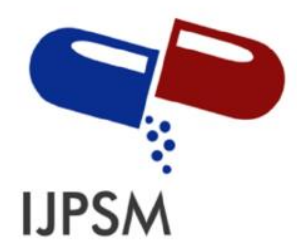

Nikam Supriya S. et al, International Journal of Pharmaceutical Sciences and Medicine (IJPSM), Vol.6 Issue. 7, July- 2021, pg. 81-101

surfactant concentration rises. Furthermore, particle size is affected by the quantity and percentage of distinct solid and liquid lipids.

\section{Drug entrapment efficiency}

The concentration of medication in the dispersion media is determined by centrifuging a specified amount of NLC dispersion and analysing the supernatant. The free drug will stay in the supernatant after centrifugation, forming NLC sediment. To calculate the concentration of entrapped drug, subtract the initial concentration of drug in dispersion from the free drug concentration. If a drug's solubility in a lipid blend is higher, the entrapment efficiency is higher, and drug escape from the matrix in the media is reduced.

$\% \mathrm{EE}=(\mathrm{Ic}-\mathrm{Fc}) \mathrm{Ic} \times 100$

Ic $=$ Initial concentration of drug in dispersion

$\mathrm{Fc}=$ Concentration of drug in supernatant

\section{Polydispersity index (pdi)}

The dynamic light scattering method can be used to determine PDI (PCS). The product's PDI describes the size distribution of Nano carriers. Formulations with PDI values less than 0.5 are homogeneous and monodisperse, while those with PDI values greater than 0.5 are non-homogeneous and polydisperse. The smaller the particle size, the less the size distribution fluctuations will be, and the lower the PDI will be. Because colloidal carrier systems are not necessarily monodisperse, a PDI value of 1 is preferred.

\section{Zeta potential}

Photon correlation spectroscopy can be used to determine zeta potential. The zeta potential is a measure of colloidal dispersion stability. Aggregation of the particles is avoided by accumulating surface charge on the particles $(30 \mathrm{mV})$. The majority of the time, lipid Nano carriers acquires a negative charge, but when mucoadhesion or BBB crossing is required, the carrier surface requires a positive charge. Surface charge is also caused by adsorption of surfactants and coating materials.

\section{Shape and morphology}

Scanning Electron Microscopy (SEM) creates images of components by scanning the surface with a focused beam of electrons, whereas Transmission Electron Microscopy (TEM) creates images by transmitting electrons through the specimen. SEM and TEM, on the other hand, disclose the surface shape and structure of particles from the inside. SEM displays the particle's surface morphology, shape, porous nature, and size, whereas TEM reveals the particle's structure from the inside and provides information on particle diameter and matrix structure. 


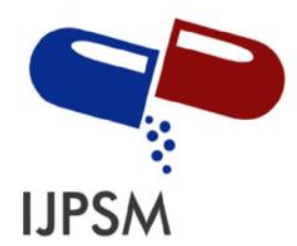

Nikam Supriya S. et al, International Journal of Pharmaceutical Sciences and Medicine (IJPSM), Vol.6 Issue. 7, July- 2021, pg. 81-101

ISSN: 2519-9889

Impact Factor: 3.426

\section{Drug- lipid (excipient) interaction}

Peaks in Fourier Transform Infrared Spectroscopy (FTIR) reveal the wave number at which specific functional groups in molecules transmit IR radiations. From $4000 \mathrm{~cm}^{-1}$ to $400 \mathrm{~cm}^{-1}$, the transmission can be measured. The FTIR method is commonly used to determine interactions between drugs and excipients/ lipids. The drug excipient interaction is demonstrated by the shifting or weakening of drug functional group peaks or the emergence of previously undetected peaks in the physical combination. Peaks of functional groups of lipids can be seen in NLCs as a drug is implanted in the matrix.

\section{Drug encapsulation in NLCs ${ }^{[36]}$}

Three methods exist for encapsulating drugs into lipid nanoparticles or NLCs. They consist of a solid solution matrix, a drug-enriched shell, and a drug-enriched core.

(1) Homogenous matrix of solid - The drug is consistently disseminated into the lipid matrix of the particles in this approach, and subsequently drug release happens via diffusion.

(2) Drug-enriched shell - The medicine is focused on the lipid nanoparticles' exterior layer or shell in this encapsulation approach. Because of the precipitation and solubilization mechanisms, this form of nanoparticle has a burst release of the medication.

(3) Drug-enriched - The saturation solubility of the medication in the lipid causes extended release in this form of encapsulation.

\begin{tabular}{|l|l|l|l|}
\hline Sr.no & Product name & Active ingredient & Manufacturer \\
\hline 1 & $\begin{array}{l}\text { Nano-lipid restore } \\
\text { CLR }\end{array}$ & $\begin{array}{l}\text { Black current seed oil containing 3 and 6 } \\
\text { unsaturated fatty acids }\end{array}$ & $\begin{array}{l}\text { Special chem. } \\
\text { (France) }\end{array}$ \\
\hline & $\begin{array}{l}\text { Nano-lipid basic } \\
\text { CLR }\end{array}$ & Caprylic / Capric triglyceride & $\begin{array}{l}\text { Dr. Kurt Richter } \\
\text { (Germany) }\end{array}$ \\
\hline 3 & $\begin{array}{l}\text { NLC deep effect } \\
\text { eye serum }\end{array}$ & Coenzyme Q10, Oligosaccharides & $\begin{array}{l}\text { Beate Johnen } \\
\text { (Germany) }\end{array}$ \\
\hline 4 & $\begin{array}{l}\text { Extra moist softener } \\
\text { Coenzyme Q10, 3 and 6 unsaturated fatty acids }\end{array}$ & $\begin{array}{l}\text { Amore Pacific } \\
\text { (Korea) }\end{array}$ \\
\hline 5 & $\begin{array}{l}\text { NLC deep effect } \\
\text { repair cream a10, Titanium dioxide, highly active } \\
\text { Beate Johnen } \\
\text { (Germany) }\end{array}$ & $\begin{array}{l}\text { Oligosaccharides } \\
\text { Table2. Marketed products containing NLCs }\end{array}$ \\
\hline
\end{tabular}




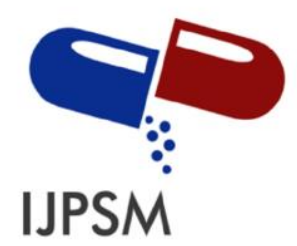

Nikam Supriya S. et al, International Journal of Pharmaceutical Sciences and Medicine (IJPSM), Vol.6 Issue. 7, July- 2021, pg. 81-101

ISSN: 2519-9889

Impact Factor: 3.426

\section{Current development of NLCs in topical delivery systems ${ }^{[38][39][40][41]}$}

1. Formulation and characterization solid lipid nanoparticles, nanostructured lipid carriers and nanoemulsions of Lornoxicam for transdermal delivery were studied by Gonullu et al (2015). Lornoxicam (LRX) solid lipid nanoparticles (SLN), nanostructured lipid carriers (NLC), and nanoemulsions (NE) were developed for the treatment of painful and inflammatory skin conditions. As solid and liquid lipids, Compritol® 888 ATO, Lanette ${ }^{\circledR} \mathrm{O}$, and oleic acid were employed. At various temperatures, solid lipid nanoparticles (SLN), nanostructured lipid carriers (NLC), and nanoemulsions (NE) were found to be physically stable for 6 months. The dominant mechanism of Fickian drug diffusion from nanoparticles and nanoemulsions was identified as Case I diffusional drug release. Nanoemulsions had the highest medication penetration rate via rat skin, followed by Nanostructured lipid carriers (NLC), Solid lipid nanoparticles (SLN), and a gel formulation. In comparison to the gel, nano formulations appeared to improve medication penetration through rat skin. ${ }^{38}$

2. Formulation of sildenafil citrate loaded Nanostructured lipid carriers and solid lipid nanoparticles by modified high-shear homogenization technique were studied by Elnaggar et al (2011). Particle size analysis, entrapment efficiency (EE) calculation, freeze-drying and reconstitution, differential scanning calorimetry, in vitro release, stability study, and HPLC analysis were among the optimization stages. With hopeful inferences for faster onset and longer drug duration, transdermal penetration of SC showed increased initial release from both Solid lipid nanoparticles and Nanostructured lipid carrier formulations, followed by regulated release. ${ }^{39}$

3. Development of Solid lipid nanoparticles (SLN) and Nanostructured lipid carrier (NLC) by using Precirol ATO 5 as the solid lipid and squalene as liquid lipid for topical psoralen delivery were studied by Fang et al (2008). The mean particle diameters of solid lipid nanoparticles (SLN) and nanostructured lipid carrier (NLC) were 300 and $200 \mathrm{~nm}$, respectively. Except for the NLC with Tween 80 and soybean phospholipids as emulsifiers, all Nanoparticulate systems had Newtonian viscosity (NLC-Tw). The NLC was used to achieve improved penetration and regulated release of psoralen administration. According to the in vitro permeation studies, NLC-Tw increased the psoralen flux by 2.8 times over a standard emulsion. ${ }^{40}$

4. Development of potential topical treatment containing Nanostructured Lipid Carrier (NLC) for Rheumatoid Arthritis (RA) was reported by Gokhale et al (2018). Quercetin (QCT) loaded NLC were prepared and evaluated for particle size distribution, polydispersity index, zeta potential analysis, in vitro drug release study. QCT-NLC gel showed pseudo plastic flow behavior with excellent texture profile parameters. In vitro activity was carried out using Complete Freund's Adjuvant (CFA) induced arthritic model. Results displayed that QCT-NLC gel appears a possible formulation system for topical delivery of QCT in rheumatoid arthritis treatment. $^{41}$

Conclusion: The application of Nano carriers to topical medication administration has ushered in a new era in drug delivery. NLCs (nanostructured lipid carriers) are chemically and physically stable systems that have 


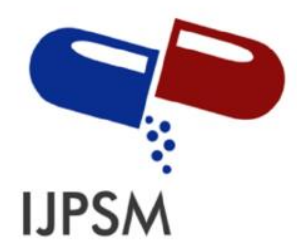

Nikam Supriya S. et al, International Journal of Pharmaceutical Sciences and Medicine (IJPSM), Vol.6 Issue. 7, July- 2021, pg. 81-101

ISSN: 2519-9889

Impact Factor: 3.426

improved medication integration and bioavailability. The industry's interest in lipid carrier systems has grown in recent years, with significant developments. Various commercial NLC formulations including medicinal and cosmetics ingredients are currently available on the market. As a wiser generation of lipid nanoparticles, NLCs are promising options for skin targeting, occlusive action, and long-term release. Because of their ease of largescale manufacture, lipid Nano carriers are attracting industrial attention. Because of its several advantages over first-generation systems, future NLC formulations may bring more affluence to the lipid carrier system.

\section{References}

[1]. Kosnik A, Szekalska M, Winnicka K. Nanostructured lipid carrier: A potential use for skin drug delivery system. Pharmacological Reports 2019; 156-166.

[2]. Ucheni O, Ogbonna G, Attama A. Nanoparticles for Dermal and Transdermal Drug Delivery. Application of Nanotechnology in Drug Delivery 2014; 194-235.

[3]. Palmer B, DE Louise L. Nanoparticle-Enabled Transdermal Drug Delivery System for Enhanced Dose Control and Tissue Targeting. Molecules 2016; 21: 1719.

[4]. Sharma G, Thakur K, Raza K, Singh B, Katare O. Nanostructured lipid carriers: A New Paradigm in Topical Delivery for Dermal and Transdermal Applications. Critical Reviews in Therapeutic Drug Carrier System. 2017; 34(4), 355-386.

[5]. Foldvari M, Gharagozloo M, Li C. Nanoparticles for Dermal and Transdermal Drug Delivery: Permeation Pathways and Applications. Handbook of Nano biomedical Research. 2014; 231-260.

[6]. Cui M, Wiraja C, Xu C. Nano-delivery systems for topical management of skin disorders. Molecular Pharmaceutics. Molecular Pharmaceutics. 2020; 1-36.

[7]. Senni R, Krishnamurthy S, Chan F. Nanoparticles for Improved Topical Drug Delivery for Skin diseases. Nanotechnology for Biomedical Applications. 2016; 274-294.

[8]. Goyal R, Macri L, Kaplan H, Kohn J. Nanoparticles and nanofibers for topical drug delivery. Journal of Controlled Release. 2015; 1-45.

[9]. Singla V, Saini S, Joshi B, Rana A. Emulgel: A new platform for topical drug delivery. International Journal of Pharma and Bio Sciences. 2012; 3(1): 485-498.

[10].Karamsetty V, Tej S, Moin A, Gowda D, Karunakar G, Patel N, Kamal S. Nano structured lipid carrier based drug delivery system. Journal of Chemical and Pharmaceutical Research. 2016; 8(2): 627-643. 


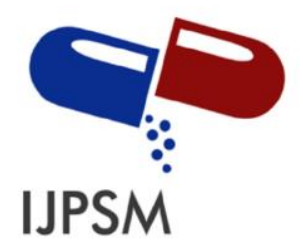

Nikam Supriya S. et al, International Journal of Pharmaceutical Sciences and Medicine (IJPSM), Vol.6 Issue. 7, July- 2021, pg. 81-101

[11].Ghasemiyeh P, Samani S. Solid lipid nanoparticles and nano structured lipid carriers as novel drug delivery system: applications, advantages and disadvantages. Research in Pharmaceutical Sciences. 2018; 13(4): 288-303.

[12].Bhaskar K, Mohan C, Lingam M, Reddy V, Venkateswarlu V, Rao Y. Development of Nitredipine Controlled Release Formulations Based on SLN and NLC for Topical Delivery: In vitro and Ex vivo Characterization. Drug Development and Industrial Pharmacy. 2008; 34: 719-725.

[13].Li Q, Cia T, Huang Y, Xia X, Cole S, Cia Y. A Review of Structure Preparation, and Application of NLCs, PNPs, and PLNs. Nano materials. 2017; 7: 122.

[14]. Bhatt A, Kumar M, Devi S, Upadhyay P, Saini V. Recent Advances in the Development of Nanostructured Lipid Carriers for the Topical Fungal Infections. Journal of Reports in the Pharmaceutical Sciences. 2020; 9(27): 1-8.

[15].Joshi M, Patravale V. Nanostructured lipid carrier (NLC) based gel of celecoxib. International Journal of Pharmaceutics. 2007; 124-132.

[16].Sharma A, Baldi A. Nanostructured Lipid Carriers: A review. Journal of Developing Drugs. 2018; 7(2): 1-12.

[17].Singh N, Chawla S. Recent Advances in Nanostructured Lipid Carriers for Biomedical Applications: A Review. International Journal of Basic and Applied Biology. 2017; 4(1): 40-44.

[18]. Mazumder S, Pavurala N. Enhanced Dissolution of Poorly Soluble Antiviral Drugs from Nanoparticles of Cellulose Acetate based Solid Dispersion Matrices. Asian Journal of Pharmaceutical Science. 2017; 12(6): 532-41.

[19].Chauhan I, Yasir M, Verma M, Singh A. Nanostructured Lipid Carriers: A Groundbreaking approach for Transdermal Drug Delivery. Advanced Pharmaceutical Bulletin. 2020; 10(2): 150-165.

[20].Puglia C, Bonina F. Lipid Nanoparticles as Novel Delivery Systems for Cosmetics and Dermal Pharmaceuticals. Expert Opinion Drug Delivery 2012; 9(4): 429-41.

[21]. Wang J, Wang H. Physicochemical Characterization, Photo-stability and Cytotoxicity of Coenzyme Q10-loading Nanostructured Lipid Carrier. Journal of Nano science and Technology. 2012; 12(3): 2136-48.

[22]. Rancan F, Vogt A. Getting under the Skin: What is the Potential of the Trans follicular Route in Drug Delivery? Therapeutic Delivery 2014; 5(8): 875-7.

[23].Gilaberte Y, Pastushenko I. Anatomy and Function of the Skin. Nano science in Dermatology. 2016; $1-14$.

[24].Maghraby GM, Barry BW. Liposomes and Skin: from Drug Delivery to Model Membranes. European journal of pharmaceutical sciences. 2008; 34(4-5): 203-22. 


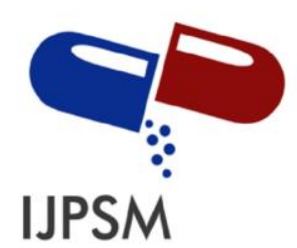

Nikam Supriya S. et al, International Journal of Pharmaceutical Sciences and Medicine (IJPSM), Vol.6 Issue. 7, July- 2021, pg. 81-101

ISSN: 2519-9889

Impact Factor: 3.426

[25].Ismail R, Basri M. Nanostructured Lipid Carriers (NLC) for Efficient Delivery of Palm Phytonutrients. Journal of Oil Palm Research. 2014; 26(3): 232-9.

[26].Müller RH, Shegokar R. 20 Years of Lipid Nanoparticles (SLN and NLC): Present State of Development and Industrial Applications. Current Drug Discovery Technologies. 2011; 8(3): 207-27.

[27].Wissing SA, Muller RH. Solid Lipid Nanoparticles for Parenteral Drug Delivery. Advanced Drug Delivery Reviews. 2004; 56(9): 1257-72.

[28].Loo C, Ismail R. Effect of Compositions in Nanostructured Lipid Carriers (NLC) on Skin Hydration and Occlusion. International Journal of Nano medicine. 2013; 8:13-22.

[29].Fang CL, Fang JY. Nanostructured Lipid Carriers (NLCs) for Drug Delivery and Targeting. Recent Patents on Nanotechnology. 2013; 7(1):41-55.

[30].Gelfuso GM, Cunha-Filho MS. Nanostructured Lipid Carriers for Targeting Drug Delivery to the Epidermal Layer. Therapeutic Delivery. 2016; 7(11): 735-7.

[31].Mokhtari M, Jaafari MR. Preparation, Characterization and Evaluation of Moisturizing and UV Protecting Effects of Topical Solid Lipid Nanoparticles. Brazilian Journal of Pharmaceutical Sciences. 2012; 48(4): 683-90.

[32]. Tichota DM, Silva AC. Design, Characterization, and Clinical Evaluation of Argan Oil Nanostructured Lipid Carriers to Improve Skin Hydration. International journal of Nano medicine 2014; 9(38): 55-64.

[33].Fang C, Fang J. Nanostructured Lipid Carriers (NLCs) for Drug Delivery and Targeting, Recent patents on Nanotechnology. 2013; (7): 41-55.

[34].Shah N, Seth A. Nanostructured Lipid Carriers for Oral Bioavailability Enhancement of Raloxifene: Design and in Vivo Study. Journal of Advanced Research. 2016; (7): 423-434.

[35]. Rizwanullah M, Amin S. Improved Pharmacokinetics and Antihyperlipidemic Efficacy of Rosuvastatin Loaded Nanostructured Lipid Carriers. Journal of Drug Targeting. 2017; (25): 58-78.

[36]. Das S, Chaudhary A. Recent advances in lipid nanoparticle formulations with solid matrix for oral drug delivery. AAPS Pharm-SciTech. 2011; 12(62): 76.

[37].Nandvikar N, Lala R. Nanostructured lipid carrier: The advanced lipid carriers. International Journal of Pharmaceutical Science and Research. 2019; 10(12): 5252-5265.

[38].Gonullu U, Uner M. Formulation and Characterization of Solid Lipid Nanoparticles, Nanostructured Lipid Carriers and Nanoemulsions of Lornoxicam for Transdermal Delivery. Acta Pharm. 2015; (65): $1-13$. 


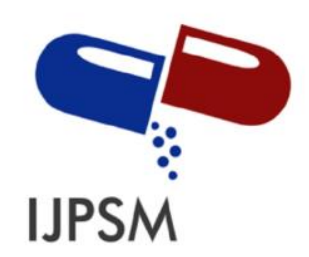

Nikam Supriya S. et al, International Journal of Pharmaceutical Sciences and Medicine (IJPSM), Vol.6 Issue. 7, July- 2021, pg. 81-101

ISSN: 2519-9889 Impact Factor: 3.426

[39].Elnaggar Y, Massik M. Fabrication, Appraisal, and Transdermal Permeation of Sildenafil CitrateLoaded Nanostructured Lipid Carriers versus Solid Lipid Nanoparticles. International Journal of Nano medicine. 2011; (6): 3195-3205.

[40].Fang J, Fang C. Lipid Nanoparticles as Vehicles for Topical Psoralen Delivery: Solid Lipid Nanoparticles (SLN) Versus Nanostructured Lipid Carriers (NLC). European Journal of Pharmaceutics and Bio pharmaceutics. 2008; (70): 633-64.

[41].Gokhale J, Khurana S. Lipid nanoparticles as vehicles for topical psoralen delivery: Solid lipid nanoparticles (SLN) versus nanostructured lipid carriers (NLC). International Journal of pharmaceutical Sciences and Nanotechnology. 2018; 11(1): 3967-3976. 